\title{
Fabrication and Excellent Dielectric Performance of Exfoliated Graphite Sheets
}

\author{
Girish M. Joshi1,*, Kalim Deshmukh¹, P.K. Jain² \\ ${ }_{1}$ Polymer Nanocomposite Laboratory, Material Physics Division, School of Advanced Sciences, \\ VIT University, Vellore-632014, TN, India \\ 2 Center for Carbon Materials, International Advanced Research Center for Powder Metallurgy and New Materials, \\ Autonomous Center of Department of Science and Technology, P. O. Balapur, Hydrabad-500 005, India.
}

(Received 04 December 2015; published online 15 March 2016)

\begin{abstract}
In the present investigation, exfoliated graphite sheets were obtained from the thermo-chemical treatment of natural graphite flakes. In this process, the graphite expands almost 300-350 times of its original volume and takes the shape of worms. These worms can be pressed or rolled into any desired shape without any binder. The exfoliated graphite sheets show excellent electrical properties i.e. high dielectric constant $\left(\varepsilon=6.374 \times 10^{7}\right)$ and comparatively low dielectric loss $(\tan \delta=138)$ across the frequency in the range $50 \mathrm{~Hz}$ to $30 \mathrm{MHz}$ and temperature in the range $40-300{ }^{\circ} \mathrm{C}$. The products developed from exfoliated graphite can have very good sealing applications in industrial areas, especially for gaskets in the automobile industries.
\end{abstract}

Keywords: Carbon materials, Dielectrics, Electrical properties, Electron microscopy.

PACS numbers: 81.05.ue, 72.80.Vp

\section{INTRODUCTION}

The Single layer graphene has attracted much attention in the past several years because of its exceptional properties such as high surface area, high mechanical strength and high elasticity [1,2]. Obtaining graphene in the form of nanoribbon sheets is of technological importance and can be utilized as a compatible medium [3]. The graphene nanoribbon sheets with an optimum size of $40 \mathrm{~nm}$ produced by chemical treatment method has been used widely for several technological applications. The shaping and yielding of the graphite is one of the key interest to explore the possibility to use this material for electrical circuits, field effect transistors and graphene based integrated circuits. It is also most suitable for several industries such as chemical, biomedical as machined and processed nano entity $[4,5]$. Numerous studies have been under taken to exfoliate graphene sheets by soincation [6], chemical route [7], photocatalytic process [8], ion based electro-chemical route [9], modular shear loading and mechanical drilling [10-12].

Herein, we developed exfoliated graphite sheets by thermo-chemical treatment of graphite flakes. The microstructure of these exfoliated graphite sheets were studied by SEM. The impedance analyzer was used to study the electrical properties of the exfoliated graphite sheets as a function of frequency in the range $50 \mathrm{~Hz}$ $30 \mathrm{MHz}$ and temperature in the range $40-300{ }^{\circ} \mathrm{C}$.

Preparation exist, and submitted manuscript should be ready for publication. Simultaneously with the content reviewing, any manuscript is checked for compliance to the stated formatting requirements. If the checking is failed, the manuscript is returned to authors for revision.

\section{EXPERIMENTAL AND MATERIALS}

\subsection{Materials}

Natural Graphite flakes were supplied by Carbotech Engineers PVT, Ltd, Jaipur, India. Sulphuric acid
(H2SO4) and nitric acid (HNO3) was purchased from Sisco Research Laboratories, PVT, Ltd., India.

\subsection{Protocol to Obtain Exfoliated Graphite Sheets}

Graphite was chemically treated before its exfoliation. There are many oxidants, which can be used for intercalation like Nitric acid (HNO3), Hydrogen peroxide (H2O2), Potassium di-Chromate (K2Cr2O7) etc. In the present study, the mixture of Sulphuric acid (H2SO4) and Nitric acids (HNO3) in a 3:1 ratio was used for graphite intercalation. Once the graphite flakes were intercalated, they were subjected to thermal shock at $1000{ }^{\circ} \mathrm{C}$ in a specially designed furnace. The thermal shock results into a very high expansion of these graphite flakes, almost 300 to 350 times the original volume of graphite flakes, depending on the process parameters.

The surface area of exfoliated graphite sheets has been obtained using (11-2370) Gemini, Micromeritics, USA, surface area analyzer at a regeneration temperature of $200^{\circ} \mathrm{C}$ for $2 \mathrm{~h}$ with $\mathrm{N} 2$ purging.

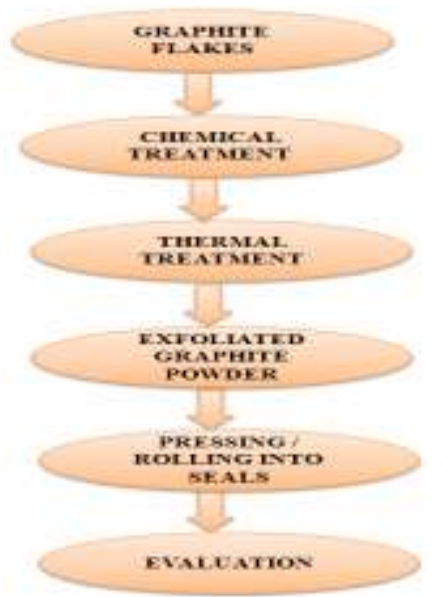

Fig. 1 - Process flow chart of exfoliated graphite product

\footnotetext{
*varadgm@gmail.com
} 


\subsection{Characterizations}

The surface morphology of the sample was analyzed using Phillips XL 30 Series field emission scanning electron microscope operating at an accession voltage of $25 \mathrm{kV}$ under vacuum $\left(10^{-5} \mathrm{mbar}\right)$.

The dielectric properties of exfoliated graphite sheets were measured using a Newton's 4th Impedance Analyzer (PSM1735, N-4L,UK) as a function of frequency in the range $50 \mathrm{~Hz}$ to $30 \mathrm{MHz}$ and temperature in the range $40-300{ }^{\circ} \mathrm{C}$.

\section{RESULTS AND DISCUSSION}

The exfoliated graphite sheet was produced from unique thermo-chemical treatment of natural graphite flakes. The process flow chart for exfoliation of graphite is shown in Fig. 1. In this process, the graphite expands almost 300-350 times of its original volume and takes the shape of worms. These worms can be pressed or rolled into any desired shape without any binder. The compression or rolling of exfoliated graphite particles give a flexible sheet which is usually named as flexible graphite foil. The sheet is having an excellent salient features such as impermeable to gases and fluids and can be used from -200 to $+500{ }^{\circ} \mathrm{C}$ in the oxidizing atmospheres and up to $2500{ }^{\circ} \mathrm{C}$ in the non-oxidizing atmosphere or vacuum. It cannot be wetted with molten glass, metals etc. It offers high anisotropy in electrical and thermal conductivities. It exhibits excellent temperature shock resistance. It does not age or creep. It is self-lubricating and can be easily cut and punched. It is resistant to radiation doses of any magnitude occurring in nuclear power stations. The obtained graphite worms are pressed and rolled into graphite sheets and tapes as shown in Fig. 2.

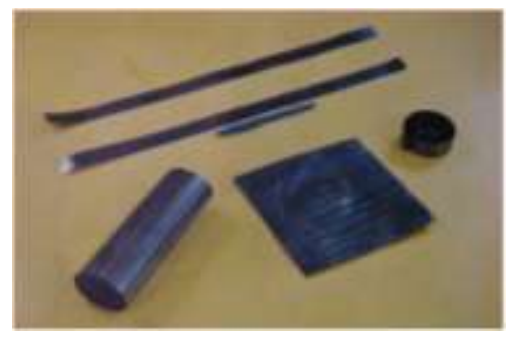

Fig. 2 - Flexible Graphite tapes and foils
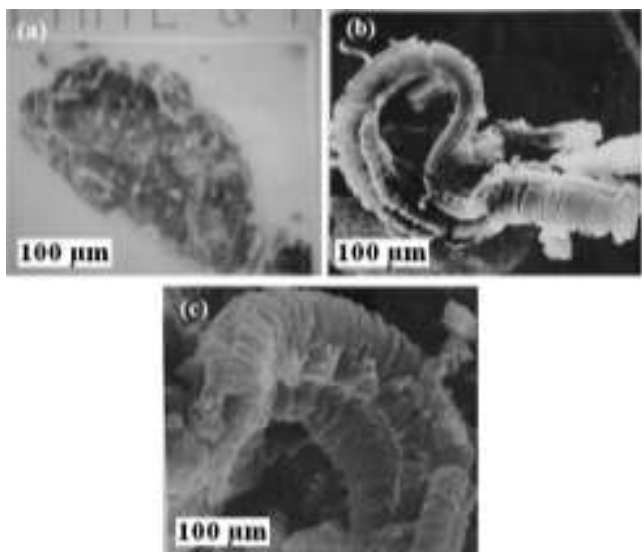

Fig. 3 - Micrographs of (a) Graphite Flakes (b, c) Exfoliated Graphite Worms
It was interesting to know the surface area of the graphite worms produced by graphite flakes. The surface area and the degree of exfoliation of graphite powders is given in Table 1.

Table 1 - Surface area and degree of exfoliation of Graphite powders

\begin{tabular}{|l|l|l|}
\hline Sample & $\begin{array}{l}\text { Degree of Exfo- } \\
\text { liation }\end{array}$ & $\begin{array}{l}\text { Active Surface } \\
\text { area (m²/gm) }\end{array}$ \\
\hline $\begin{array}{l}\text { Graphite Flakes } \\
(+40)\end{array}$ & - & 1.5 \\
\hline $\begin{array}{l}\text { Exfoliated } \\
\text { Graphite }\end{array}$ & 350 & 45.5 \\
\hline
\end{tabular}

It can be seen that the surface area of exfoliated graphite sheet was increased upon exfoliation. The SEM micrographs of worms shows graphite expansion in c-direction only and in a-direction there was no change. At higher magnifications of graphite worms one can observe the honeycomb structure of graphite layers. A very high expansion in the c-direction of graphite flakes, was observed through SEM images as shown in Fig. 3 (a-c). The microstructure of worm was like a honeycomb structure or Chinese accordion.

Recently, the electrochemical impedance spectroscopy technique was employed to distinguish between graphene, graphene oxide and different graphene based materials $[13,14]$. In the present study, the dielectric properties of exfoliated graphite sheets were carried out and the results are depicted in Fig. 4.
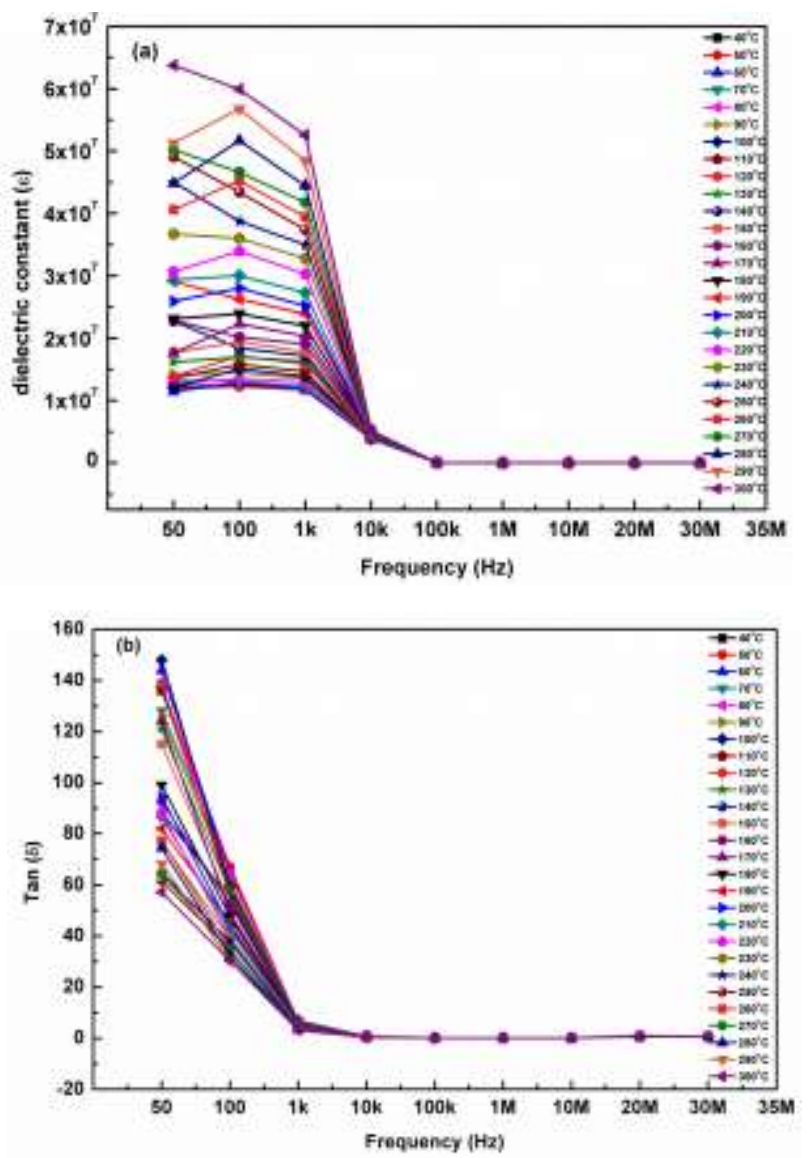

Fig. 4 - Dielectric Constant (a) and Dielectric Loss (b) of G-sheets as function of temperature 
The exfoliated graphite sheets show very high dielectric constant $\left(\varepsilon=6.374=10^{7}\right)$ and comparatively low dielectric loss $(\tan \delta=138)$ as a function of frequency in the range $50 \mathrm{~Hz}$ to $30 \mathrm{MHz}$ and temperature in the range $40-300{ }^{\circ} \mathrm{C}$. The dielectric loss can be optimized further by chemically modifying the exfoliated graphite sheets for its implementation in industrial applications. Hence, such materials with superior dielectric properties can be useful in electrical and electronic industry for capacitors, supercapacitors and/or embedded capacitors applications.

The exfoliated graphite sheet was produced from unique thermo-chemical treatment of natural graphite flakes. In this process the graphite expands almost 300350 times of its original volume and takes the shape of worms. Therefore, the products developed from this process are not only retaining the excellent inherent

\section{REFERENCES}

1. K.S. Novoselov, A.K. Geim, S.V. Morozov, D. Jiang, Y. Zhang, S.V. Dubonos, Science 306, 666 (2004).

2. A.K. Geim, Science 324, 1530 (2009).

3. Z.S. Wu, W. Ren, L. Gao, B. Liu, J. Zhao, H.M. Cheng, Nano Res. 3, 16 (2010).

4. J. An, Y.Q. Gou, C.X. Hu, F.D. Yang, C.M. Wang, Mater. Sci. Eng. C 33, 2827 (2013)

5. X.H. Hu, X.H. Ma, H.P. Tan, D. Li, Micro Nano Lett. 8, 277 (2013)

6. K. Deshmukh, S.M. Khatake, G.M. Joshi, J. Polym. Res. 20, 286 (2013).

7. K. Deshmukh, G.M. Joshi, Polym. Test. 34, 211 (2014).

8. H. Liu, Y. Su, Z. Chen, Z. Jin, Y. Wang, J. Hazard. Mater. properties of graphite but also complex processing free from carbonizations and graphitization can be avoided. Moreover, the products are flexible in nature unlike brittle as in the case of synthetic graphite. The exfoliated graphite sheet show excellent electrical properties such as high dielectric constant and comparatively low dielectric loss. Hence, such materials can be useful in different industries such as in electronic, electrical and nuclear industry to prepare prototype products.

\section{ACKNOWLEDGEMENTS}

The authors wish to thank Naval Research Board, Defence Research and Development organization (NRBDRDO), New Delhi for providing facility for electrical characterization under project No 259/Mat./11-12.

266, 75 (2014)

9. Y. Yang, F. Lu, Z. Zhou, W. Song, Q. Chen, X. Ji, Electrochim. Acta 113, 9 (2013).

10. M. Mohammadi, A. Farajpour, M. Goodarzi, H. Shehni Nezhad Pour, Comp. Mat. Sci. 82, 510 (2014).

11. V. Vijayaraghavana, A. Garga, C.H. Wong, K. Tai, S.S. Mahapatra, Measurement 50, 50 (2014).

12. G.M. Joshi, K. Deshmukh, J. Elect. Mat. 43, 1161 (2014).

13. E. Casero, A.M. Parra-Alfambra, M.D. Petit-Domínguez, F. Pariente, E. Lorenzo, C. Alonso, Electrochem. Commun. 20, 63 (2012).

14. B. Alessandra, P. Martin, Electrochem. Commun. 26, 52 (2013). 